\title{
Measuring Interruption: Syntactic and Contextual Methods of Coding Conversation
}

\author{
DINA G. OKAMOTO* \\ University of California, Davis \\ LISA SLATTERY RASHOTTE \\ University of North Carolina, Charlotte \\ LYNN SMITH-LOVIN \\ University of Arizona
}

\begin{abstract}
In this paper we focus on a long-standing debate surrounding the measurement of interruptions in conversational behavior. This debate has implications for conversational analysts interested in turn-taking structures, researchers interested in close relationships who interpret them as an exercise of power, and group processes researchers studying status-organizing structures. We explore two different measurements of interruptions: (1) a syntactic measurement that operationalizes an interruption as simultaneous talk initiated more than two syllables from the end of a current speaker's sentence, and (2) a more contextual measurement that takes into account situational factors such as the current speaker's intentions and the content of what both speakers say when judging whether a speech act is an interruption. We coded transcripts from 86 task group discussions using West and Zimmerman's (1983) syntactic criteria and Murray's (1985) context-sensitive method for identifying interruptions. Factor analyses found a one-factor solution, an indication that both measurements capture the same underlying construct. Confirmatory factor analyses identified more subtle variations, however, suggesting that gender and subcultural differences affect how coders construe interruptions.
\end{abstract}

The structure of conversation has been the central interest of scholars in several research traditions, including those who study the structure of talk itself, those who focus on what conversation reveals about personal relationships, and those who study task group discussions to reveal how work is organized and how decisions are made. Early conversation analytic researchers focused on a turn-taking model of conversation, arguing that talk is organized in such a way that one person holds the floor at any one time (Beattie 1980; Duncan 1972, 1973; Sacks, Schegloff, and Jefferson 1974). Researchers soon turned their attention to disruptions of turn-taking norms and how they are distrib-

* The authors participated equally in the construction of this paper; therefore the names are presented alphabetically. The research was supported by National Science Foundation Grant SBR-9411157 to Lynn Smith-Lovin, Principal Investigator. Direct correspondence to Dina G. Okamoto, Department of Sociology, University of California-Davis, 1282 Social Sciences and Humanities Building, Davis, CA 95616. uted in everyday conversation. Zimmerman and West's (1975) influential paper on the patterns of interruptions in conversations among men and women spawned an active interest in the study of interruptions and gender, in part because of its conclusion that women disproportionately receive interruptions from men and that these interruptions are displays of dominance and control stemming from societal gender inequality. Since these fruitful beginnings, no other conversational behavior (except possibly the overall amount of participation) has received as much attention as the interruption. ${ }^{1}$

In the last 25 years, researchers interested in a variety of substantive topics have used

\footnotetext{
${ }^{1}$ See James and Clarke (1988) and Aries (1996) for excellent reviews of the interruption and gender literature. The conclusions of this literature have shifted rather markedly since the original paper by Zimmerman and West (1975), but the insight that interruptions may indicate something deeper about the structure of relationships within the larger society has remained important.
} 
insights from conversation analytic research by focusing on interruptions, overlaps, backchannel cues, and other turn-taking structures. Those with an interest in personal relationships have used interruptions as indicators of power, control, and identity (Kollock, Blumstein, and Schwartz 1985; Stets and Burke 1996). Researchers who study the dynamics of discussion in task-oriented work groups have used interruptions as evidence of status-organizing processes within those groups. Expectation states theory, in particular, conceptualized deviations from turn-taking norms as cues during interaction that group members used to form expectations about task performance. The idea was that these cues and the expectations to which they led determined stable group interaction orders. Specifically, research in the group processes tradition has examined how diffuse status characteristics (Okamoto and Smith-Lovin 2001; Smith-Lovin and Brody 1989; Smith-Lovin and Robinson 1992) and formal authority positions (Gibson 1998; Johnson 1994) have affected the power and prestige order.

Given the continuing interest in interruptions in these three traditions-conversation analytic, close relationships, and group processes-we focus here on a long-standing debate surrounding the measurement of interruptions. We explore two different kinds of measurements that have been proposed for interruptions that occur in conversation. The first measurement type is based on syntactic structure: a speech act is considered an interruption if it is initiated more than two syllables away from a possible turn-transition space. The second type is situational in the sense that contextual cues affect what one identifies as an interruption. This latter conception of interruption also might be viewed as cultural because cultural rules deem when it is appropriate for another speaker to take the floor and when that taking is inappropriate (an interruption).

Most group processes research on interruptions has used only one type of measurement, namely Zimmerman and West's (1975; West and Zimmerman 1983) syntactic coding. Unlike the original conversation analytic research, group processes studies of conversational behavior tend to rely on larger sam- ples of talk, systematic blind coding of transcripts, and statistical methods to examine the effects of social structural positions, such as gender and authority, on conversational outcomes. Therefore we ask: Does this syntactic coding correspond to interruptions as perceived by the group members participating in the discussion? If the two constructs are somewhat different, what are the implications for research findings?

To address these questions about the measurement of interruptions, we use smallgroups data and code transcripts, identifying interruptions with both types of measurement. We then use exploratory factor analysis and confirmatory factor analysis to discover whether the two types of measurement are capturing the same underlying construct. Below, we explain these two types of measurement in detail.

\section{MEASURING INTERRUPTIONS}

To understand the dynamics of conversation, it is useful to refer to interaction as organized into turns. Sacks et al. (1974) developed a model of turn-taking in conversation which specified a set of rules ${ }^{2}$ regarding appropriate turn-taking places. A possible turn-transition space comes at the end of a completed phrase or sentence. Because the possibilities of talk are constrained by the model, three options are available when turn-transition spaces occur: the current speaker may end his or her turn at talk by addressing a new speaker, another speaker may enter the conversation, or the current speaker may continue. Sacks et al. (1974) observed that the timing of transitions is usually unproblematic and the alter-

\footnotetext{
2 The use of the metaphorical term rules has led some researchers to place an unfortunate emphasis on interruptions as "violations" which are inherently negative or indicate dominance. Many conversational analysts and researchers in related areas have attempted to counter this interpretation by pointing out that interruptions can have positive conversational functions (Bennett 1981; Edelsky 1981; Murray 1985; Smith-Lovin and Brody 1989; Tannen 1984; see related literature summarized by Aries 1996:84). Maynard and Clayman (1991) and Schegloff (2000) explicitly point out that turn-taking mechanisms are not rules in a strict sense, but instead are procedures that conversants use (most often collaboratively) for achieving the "one speaker at a time" alternating feature of conversation.
} 
nation of speakers is achieved with little or no gap; this suggests that the process is typically smooth and cooperative, and requires the interactional work of all conversants.

\section{West and Zimmerman's Syntactic Measurement of Interruptions}

Building on the perspective of Sacks and his colleagues, Zimmerman and West (1975) distinguished between two main types of simultaneous speech (in which one speaker begins to talk while another is already in progress) that occur in conversation: overlaps and interruptions. When simultaneous speech occurs close to a possible transition point, this type of conversational behavior is referred to as an overlap. They argued that, in this case, the transition is anticipated by the new speaker, and overlapping speech results from speaker transition error. In other words, a new speaker enters the conversation because he or she anticipates that the current speaker is finished with his or her talk, but the timing is a bit off: the new speaker enters the conversation just before the current speaker arrives at a possible transition place. An overlap includes those instances in a conversation when (1) a speaker gives a minimal response (e.g., "yeah," "mm-hmm") that occurs simultaneously with the current speaker's talk, or (2) two speakers say the same thing at the same time, showing agreement or "thinking along the same wavelength." This type of conversational behavior was not viewed as an intrusion into another's turn at talk, but simply as an inevitable product of the turn-taking model itself. Often, overlaps are supportive or facilitative in nature (Tannen 1986, 1994; West and Zimmerman 1983).

Operationally, Zimmerman and West (1975) coded an overlap when the new speaker starts to speak during the last syllable of the first speaker's utterance. To illustrate, we provide examples from the transcripts ${ }^{3}$ in our study in which undergraduate students acting as mock jurors attempt to reach a consensus about a recommended sentence for a perpetrator in a criminal case.

\footnotetext{
${ }^{3}$ In our transcripts, one slash (/) indicates an overlap; a double slash $(/ /)$ indicates an interruption.
}

In lines 90 and 91 from the excerpt below, Speaker B and Speaker C simultaneously speak for one syllable: "fine" and "yeah" are spoken at the same time. This is considered an overlap according to Zimmerman and West's criteria.

\begin{tabular}{|c|c|c|}
\hline 90 & $\mathrm{C}:$ & But he's / fine. \\
\hline 91 & B: & $\begin{array}{l}\text { /Yeah, I was // gonna say } \\
\text { that. }\end{array}$ \\
\hline 92 & D: & $\begin{array}{l}\text { //What if he did? So he } \\
\text { didn't hit him hard enough? }\end{array}$ \\
\hline
\end{tabular}

West and Zimmerman (1983: 104) defined interruptions operationally as "incursions that are initiated more than two syllables away from the initial or terminal boundary of a unit-type." In simpler terms, an interruption can be detected when a deep intrusion occurs more than two syllables away from a possible turn-transition space. This definition is based on syntactic structure and is theoretically embedded in the turntaking model. In the excerpt above, we see that in line 92, Speaker D interrupts Speaker B. According to West and Zimmerman, beginning to talk when Speaker B is in the middle of a sentence is considered an interruption because Speaker B is more than two syllables away from the end of his or her phrase or sentence.

West and Zimmerman argue that these "deep incursions" of two or more syllables cannot be explained as errors in timing that are produced by the constraints of the turntaking model; instead they are violations that have the potential to disrupt the current speaker's turn. An interruption is not only considered a violation of the turn-taking model, but some also interpret it as an exhibition of dominance and control in face-to-face interaction (Kollock et al. 1985; Octigan and Niederman 1979; West 1979; West and Zimmerman 1983). Other researchers, however, have pointed out that there are various types of interruptions, some of which can be facilitative (Covelli and Murray 1980; Goldberg 1990; Kennedy and Camden 1983; Murray 1987; Natale, Entin, and Joffe 1979; Smith-Lovin and Brody 1989; Tannen 1986). Regardless of content and intent, however, an utterance is coded as an interruption if it meets the structural criterion of simultane- 
ous speech at least two syllables away from a possible speaker transition point.

\section{Murray's Context-Sensitive Cultural Measurement}

Murray (1985) has taken issue with the turn-taking perspective of Sacks et al. (1974). According to Sacks et al., turns are both continuous and exclusive, such that only one person has the right to speak at any one time. Interruptions therefore are a violation of speaker rights. Murray, citing Edelsky (1981), argued that conversants themselves, when listening to recordings of their own conversations, cannot invariably identify whose turn was in progress. Particularly in groups of three or more, simultaneous speech occurred often and at great length without being apparently problematic.

Thus it is no surprise that Murray (1985, 1987; Murray and Covelli 1988) also criticized West and Zimmerman's operationally precise definition of an interruption, arguing that it fails to capture the meaning of conversational acts for participants. He pointed out that speakers pay attention to many factors when judging whether an interruption has occurred. For example, a conversant might take into account the intentions of the person already speaking and the one who follows, the content of what both speakers say, how long someone has been talking, and whether someone else has a particular claim to speak on the issue. Murray (1985:33) stated that "there are no absolute syntactic or acoustic criteria available either to those involved in conversing or those analyzing" (author's emphasis). In other words, he argued that simply counting the number of syllables where simultaneous speech occurs in conversation cannot determine what is or is not an interruption; contextual cues are an important part of the perception of overlapping speech as a violation of speaker rights.

Murray relied heavily on Basso's (1974) notion of a "completion right" in determining what people will regard as an interruption. A completion right is the speaker's right to finish his or her thought, and is determined by many situational factors. Completion rights can be affected by speaking times (how long and how often the speaker has spoken), how many points the speaker has made, whether the speaker or others have special claims to be heard (such as defending against attack, answering questions posed to him or her, or having special expertise on the topic), and subsequent repairs to previous violations. Further, Murray argued that the seriousness of violations of a speaker's completion right is variable: some are more severe and more noticeable than others. He described the following situations, beginning with the most severe:

1. Cutting the speaker off before he or she has made his or her first point of the conversation;

2. Cutting the speaker off before he or she has made the first point of a turn;

3. Cutting the speaker off in mid-clause after the first point of a turn;

4. Beginning to speak during a pause or other turn ending signal.

Below, using examples from the transcripts in our study, we provide examples of the variation in the seriousness of interruptions. In the following excerpt, Speaker C cuts off Speaker A's speech before A can make his first point in the conversation in line 1 . This demonstrates the most serious violation according to Murray.

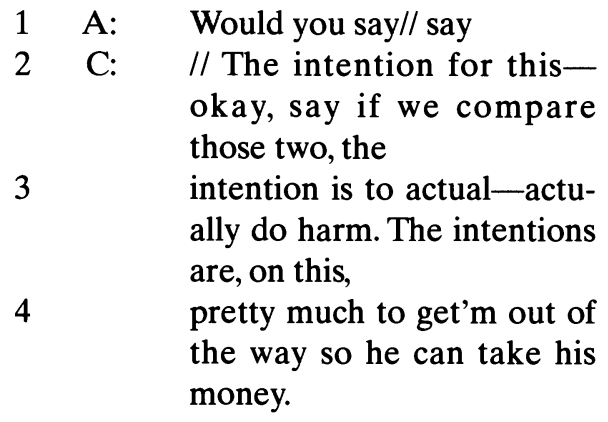

The next excerpt shows the second most severe form of interruption. Speaker A's talk is cut off by Speaker $C$ in line 13 before she makes her first point in the speaking turn.

11 C: And, I know if somebody put me away, (laughs) I would be pretty

12 pissed off.

13 A: But you don't think you // give up your right 
14 C: // And especially (when you)

$15 \mathrm{~A}$ : to walk around on the streets if you do somethin' like that? I don't

16 think-If I did somethin' like that I really don't think that I would deserve, the right, to, be on the streets, or I think / (whatever)

The third form of interruption occurs when one speaker cuts off another in midclause after he or she has made at least one point in a speaking turn. In the excerpt below, Speaker $\mathrm{C}$ is interrupted by Speaker $\mathrm{A}$ in mid-clause in line 24 while discussing the number of years for sentencing.

\begin{tabular}{|c|c|c|}
\hline 21 & A: & $\begin{array}{l}\text { I'd say, seventeen years with } \\
\text { parole. }\end{array}$ \\
\hline 22 & $\mathrm{C}:$ & $\begin{array}{l}\text { That way, if prison's rea } \\
\text { not helping him, then we } \\
\text { be keeping }\end{array}$ \\
\hline 23 & & $\begin{array}{l}\text { him out. I mean, sevente } \\
\text { years is a pretty good chun }\end{array}$ \\
\hline & & $\begin{array}{l}\text { I mean if } \\
\text { he hasn't changed// by th } \\
\text { time, }\end{array}$ \\
\hline & A: & //If he's twenty-five. \\
\hline
\end{tabular}

In the least severe form of interruption, a person begins to speak during a pause or what appears to be the end of a clause, or anywhere that is perceived to be a "turn signal." In line 42 , Speaker C begins to speak after a two-second pause, after having allowed A to continue after one three-second pause earlier in the same utterance.

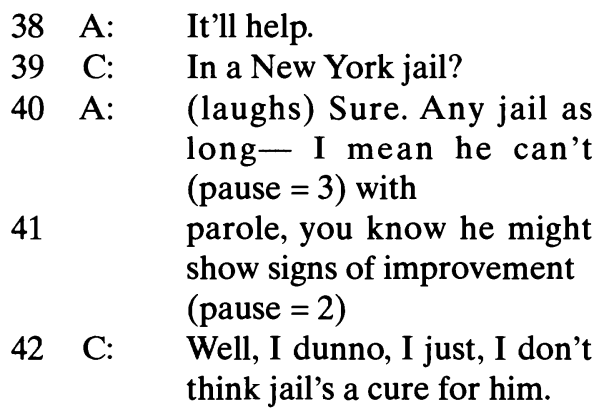

To examine the relationship between simultaneous speech and observers' definitions of interruptions, Murray asked judges to code conversations for interruptions, using information from the content of the talk both before and after the speaker transition. We call these judges "culture experts" because they are drawn from the same subcultural milieu as the speakers and presumably understand the culturally appropriate rules of interaction. Murray found that according to culture experts, not all instances of simultaneous speech are interruptions: simultaneous speech is neither necessary nor sufficient to define an interruption. He argued that purely structural criteria (e.g., number of simultaneous syllables) are not enough to determine which speech acts constitute interruptions.

Although Murray conceded that it is more difficult to study interruptions without strict criteria such as counting syllable overlap, he argued that it is necessary to identify what is intended and understood in order to accurately and appropriately gauge what is operating as an interruption in conversation. This point has important implications for the developing research literature because researchers use interruptions as indicators of power and prestige orders within task groups. Coding these large bodies of conversation typically requires an efficient, reliable definition that can be applied systematically. Yet if the material we are coding does not correspond to what interactants perceive, the variable may not represent what we have been assuming it does.

\section{Variations Among Subcultures}

Using open-ended subjective cultural criteria to identify interruptions is problematic not only because of the increased amount of measurement effort required, but also because the criteria used for identifying interruptions may not be the same for all people. For example, cultural or interethnic differences as well as regional differences in the United States may contribute to different interactional styles and subsequent differences in the way people view certain types of speech patterns (Albert 1964; Basso 1979; Cohen et al. 1999; Collier 1991; Hecht, Collier, and Ribeau 1993; Riesman 1974). For example, Tannen $(1984,1986)$ observed a high-involvement style used by New Yorkers, which is characterized by faster speech, 
abrupt topic shifts, and avoidance of pauses between turns. This conversational style contrasts with a slower-paced, high-considerateness style, which conforms to the rules of politeness and is often used by midwesterners (Lakoff 1973; Tannen 1984).

Men and women also may view conversational behavior differently because of their gendered experiences. Maltz and Borker (1982) and Tannen (1986, 1990a, 1990b) argued that girls and boys learn how to use language in different ways through their play activities in same-sex groups. Boys learn to maintain an audience and assert their own opinions; girls learn to create relational closeness by exchanging information and confidences (Goodwin 1980; Lever 1978; Maltz and Borker 1982). Thus, school-age children acquire gender-specific "cultures" that carry over into their adult lives (Thorne and Luria 1986). Consequently men and women bring different assumptions and rules to a face-toface conversation.

This theoretical perspective conceptualizes cross-sex communication as cross-cultural communication. Women's talk is viewed as more friendly, cooperative, and relational. For example, women tend to do more work to facilitate the flow of conversation (Fishman 1983) and exceed men in positive reactions (Aries 1996; Carli 1989; Strodtbeck and Mann 1956). Men's talk is more dominant, more directive, and less supportive. For instance, men are more likely to challenge or dispute another speaker's utterances, to offer no response or acknowledgment to another speaker's comments, and to change the topic of conversation (Fishman 1983; West and Garcia 1988; Zimmerman and West 1975). ${ }^{4}$ Specifically in regard to interruptions, Chambliss and Feeny (1992) find that men have a more positive view of interruptions than women and therefore are more likely to engage in this behavior. According to this gender culture perspective on conversational behavior, male and female culture experts may bring different assumptions and cultural

\footnotetext{
${ }^{4}$ We do not wish to overdraw the contrast between male and female speech styles. Most researchers have found that men and women are quite flexible in their speech styles, and are capable of using either style competently when the situation requires it (Garcia 1991).
}

rules when both observing and participating in a conversation.

We contribute to the interruptions measurement debate by finding out if the two types of measurement presented by West and Zimmerman and by Murray capture the same underlying construct. In addition, we are interested in whether the coders' gender or conversational style (due to other subcultural differences) has any effect on perceptions of what is and is not considered an interruption. ${ }^{5}$ The issue of subcultural differences in conversational cultures (whether generated by regional, stylistic, or gender differences) complicates the potential for using culture experts to code interruptions in large bodies of conversation. Therefore we explore the issue of how subcultural differences, if they exist, are related to Murray's (1985) seriousness gradient. It is reasonable to hypothesize, for example, that coders will be more likely to agree on the more serious violations of speaker rights, but that cultural rules will become more varied for less serious intrusions.

\section{DATA AND MEASUREMENT}

To examine conversational behavior in a group setting (which Murray argues is the most problematic for the turn-taking model), we use data collected during the 1991-1992 and 1993-1994 academic years at a large public university. We found no significant differences in the patterns of results from the two collection times (i.e., there was no statistical interaction between year and any other effect); thus we combine data from the two time periods in the analyses reported here. We recruited 264 undergraduate students106 male, 158 female -from sociology classes and paid them $\$ 10$ each for participation.

Each subject read one of three stimuli sets that described an actual criminal case from the State of New York. The subjects also were presented with transcripts from video-

\footnotetext{
${ }^{5}$ We did not test for cultural differences based on race, ethnicity, or nationality because the subjects and coders in our study were white and ethnicity was not recorded. We chose to conduct task group discussions that were largely homogeneous on all characteristics other than gender to reduce variation in status characteristics that could mask the main effects of interest in the original design.
} 
taped statements of the convicted perpetrator and the victim of the crime. Each subject was asked to record his or her recommended sentence for a confessed criminal. (For a highly detailed description of the design and stimuli, see Tsoudis and Smith-Lovin 1998.) The subjects then participated in a mock jury session with one to three other students. Eighty-six mock jury sessions (21 two-person groups, 38 three-person groups, 27 four-person groups) were conducted and videotaped. Each group was instructed to reach a consensus and was allowed to interact until such a consensus was reached. Groups took varying lengths of time to reach their decisions, ranging from under 10 minutes to 45 minutes. Graduate research assistants transcribed the videotapes using a subset of conventions developed by Gail Jefferson (see Atkinson and Heritage 1984). ${ }^{6}$

Following West and Zimmerman (1983; Zimmerman and West 1975), the videotapes were coded for interruptions using syntactic criteria. A research assistant blind to the hypotheses of the study coded instances of interruptions on transcripts from each session as she watched the videotapes. In this data set, the West-Zimmerman coding scheme yielded 254 interruptions in 8,888 utterances. Coding error is possible when this coding scheme is used, but it is minimal because the criteria are purely syntactic. We minimized any error, however, by having a research assistant and one of the authors review the interruptions coded on the transcripts. In cases of disagreement, the assistant and author discussed and resolved the discrepancies.

Interruptions also were identified using a culture expert method, which we believe captures many of the more complex situational elements that Murray (1985) argued determine whether a speech act is interpreted as an interruption. We instructed eight undergraduate coders (four men, four women), drawn from the same population as the origi-

\footnotetext{
${ }^{6}$ The transcripts did not include the level of detail needed for a complete conversational analysis, but instead used the conventions of that tradition to record information about pauses, overlapping speech, and other characteristics relevant to the research questions in the group processes tradition that were the focus of the original study.
}

nal subjects, to independently watch the videotapes of each group and mark the transcript in any place where they believed an interruption had occurred. We did not define an interruption for the coders; we told them that we were interested in what they thought interruptions sounded like. Coders were allowed to stop and rewind the tape at any point, and were instructed to use their best judgment throughout the process.

We believe that undergraduates are culture experts because they come from the same milieu as the group participants and therefore presumably understand culturally appropriate rules of interaction. The culture expert method captures the average person's conception of an interruption and attempts to address Murray's concerns about the importance of context in determining perceptions of conversational behavior. If we apply the most general criterion for defining an interruption using the culture expect method-counting all instances where at least one person coded a speaker transition as an interruption-we find that our data contain 2,368 interruptions. The more conservative criterion-counting a transition as an interruption when five or more (a majority) of the coders considered it an interruptionproduced 256 interruptions in the 8,888 utterances. This latter criterion does not support Murray's implicit contention that the syntactic approach will introduce problems because of overcoding; in fact, the cultural/contextual approach produced about the same number of interruptions as the West-Zimmerman syntactic coding scheme.

To clarify the two types of measurement for interruptions, we offer examples from our data showing instances where the culture expert disagreed with the coder trained in the West-Zimmerman technique. In the following excerpt, the interruption is marked in the transcript using the West-Zimmerman coding scheme: Speaker C interrupts Speaker A in mid-sentence, five syllables away from a possible turn-transition point. This is one instance where none of the culture experts marked this particular utterance as an interruption in the transcript, even though the West-Zimmerman coder did so. The culture experts may have believed that Speaker C was engaging in a facilitative speech act, 
helping Speaker A with his or her thoughts, and therefore did not mark Speaker C's utterance as an interruption.

55 A: Yeah, he doesn't remember that // he was standing there.

56 C: // 'cause they told him that he had been shot.

In the next excerpt, a culture expert coded an interruption but the WestZimmerman coder did not. This example contains no overlap in speech; we suspect that the culture expert coded this particular utterance as an interruption because he or she believed that Speaker A had not finished making a point when Speaker D began to talk.

92 A: If you're gonna put him in jail for fifteen years, it's not gonna help'm much more. You know?

93 D: Yeah, I (agree),

94 A: (If you agree that he)

95 D: but I don't think life.

96 A: But if you agree he should go to jail, //

97 D: // Oh I totally think he should go to jail.

We turn now to a discussion of our models and the methods that we used to test them.

\section{CONCEPTUAL MODELS}

We are interested in testing four theoretical/conceptual models. In Figure 1, the WestZimmerman syntactic measurement and Murray's culture expert measurement capture the same underlying construct. This model contains one underlying concept: the violation of speaker rights when one speaker takes the floor from another in violation of cultural rules. This concept may be measured in a variety of different ways, such as analyzing the conversational structure or asking different types of culture experts when they believe a violation has taken place. These measures will contain some error. In fact, the statistical models that we describe below can assess with the same degree of validity whether the indicators are related to the underlying construct and whether they contain the same degree of measurement error. The important feature in Figure 1, however, is the relationship to a single underlying construct. In contrast, Figure 2 shows a model where the two measurements capture different underlying constructs. This figure corresponds to Murray's argument that the syntactic definition fails to capture the same contextual understanding that actual conversants perceive.

In the model shown in Figure 3, the male undergraduate culture experts perceive

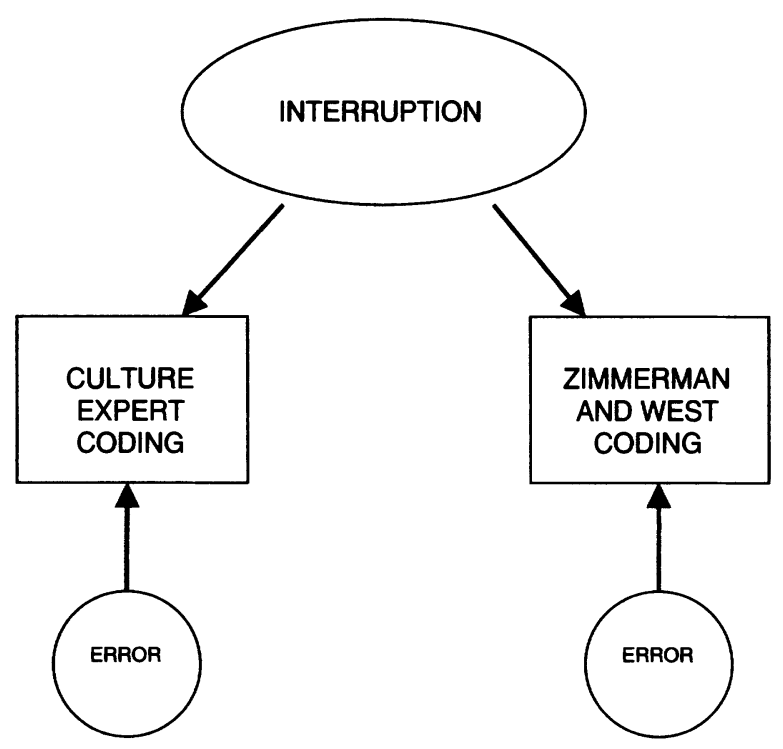

Figure 1. One-Factor Model 


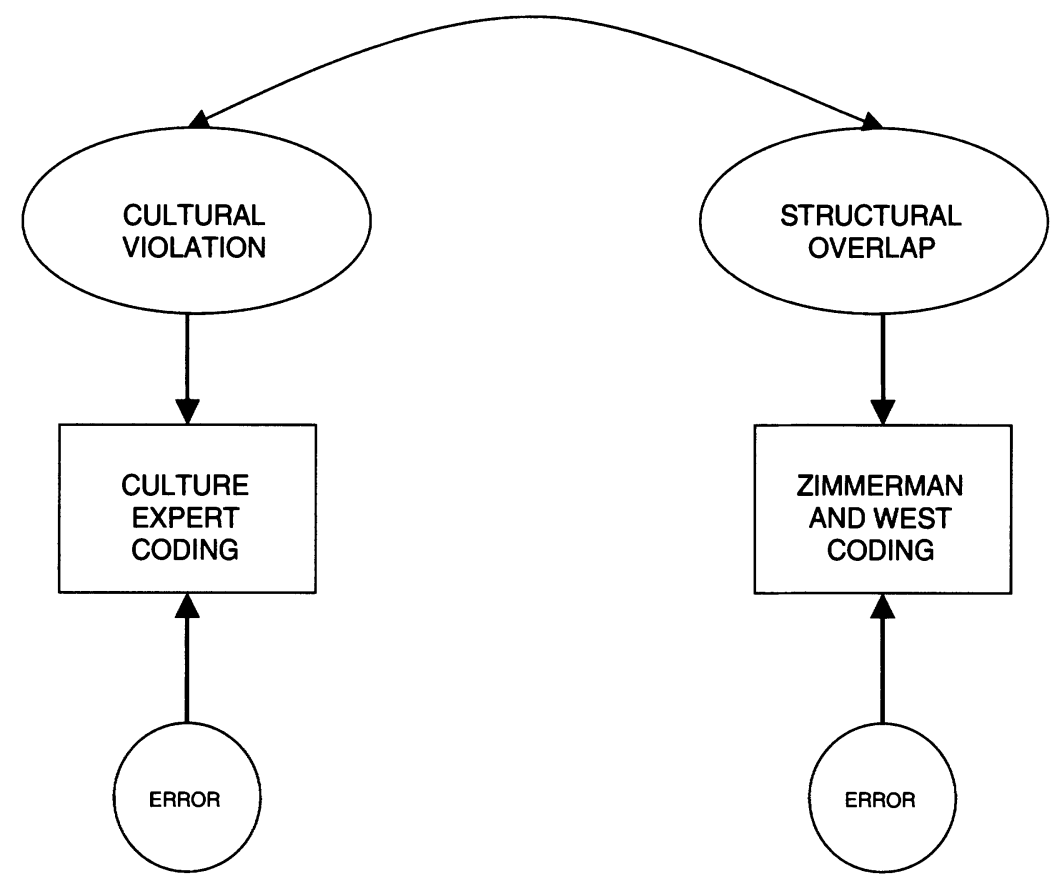

Figure 2. Two-Factor Model

interruptions so differently from female Zimmerman) capture different underlying undergraduate culture experts that the three constructs. This model represents the gender conceptions of interruptions (male culture culture argument made by Tannen (1990b) experts, female culture experts, West- and others, who suggest that men and women

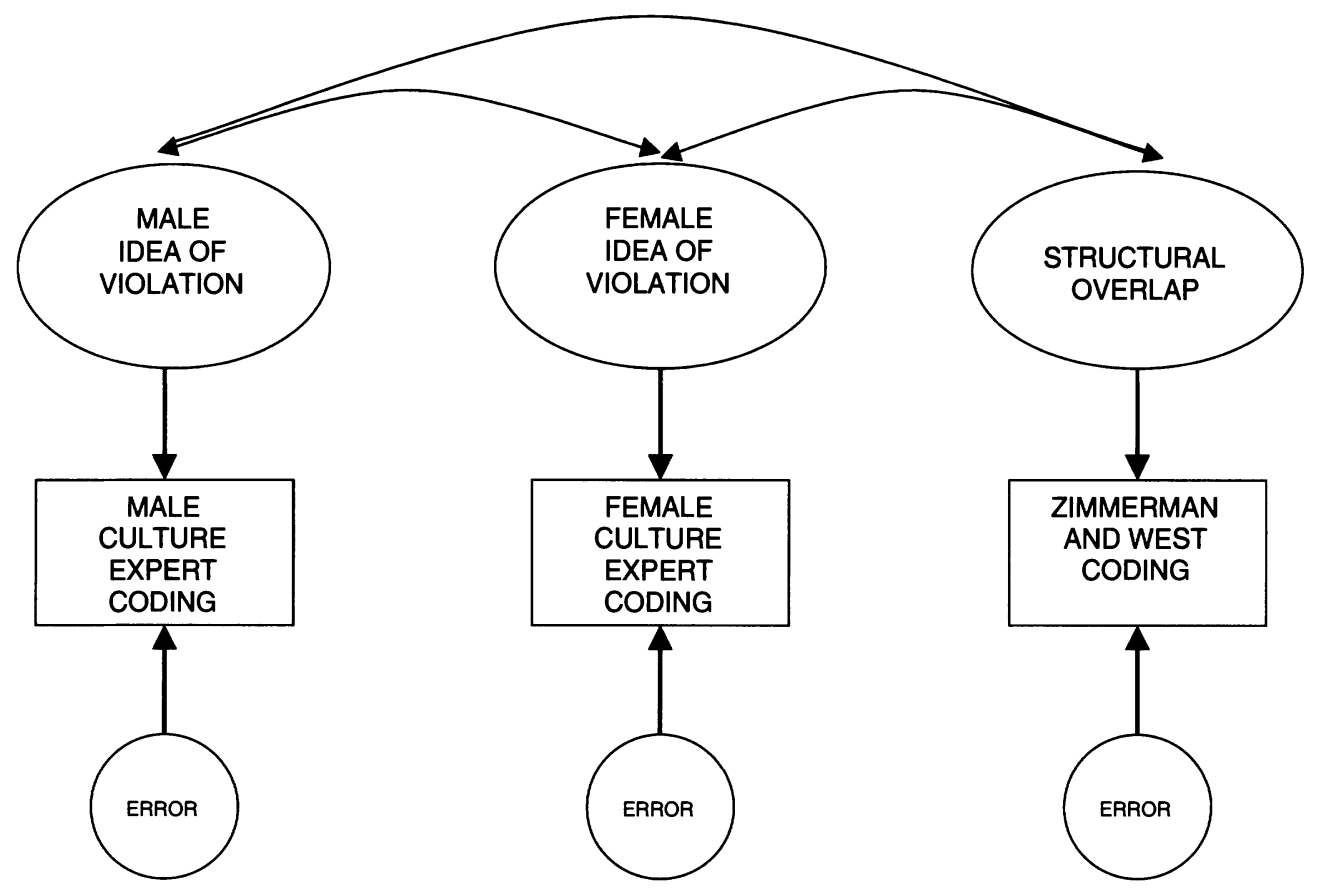

Figure 3. Gender Model 
view the nature of conversation rather differently and therefore interpret interactions in noncomparable ways.

In addition, we test a model shown in Figure 4 which posits that there are different types of culture experts: one who has low sensitivity to interruptions (and coded them infrequently) and another who has high sensitivity to interruptions (and coded them frequently). This model is based on the idea that regional or other subcultural differences in language may contribute to differences in the way culture experts identify interruptions. These differences correspond to the highinvolvement and high-considerateness types discussed by Tannen (1984). In this case, we are dealing with the perception of interruption rather than the frequent use of interruptions in conversation. We assume, following Tannen (1984), that people who use interruptions frequently also would be less likely to view other people's simultaneous speech as a violation of norms, but would instead view such speech as a lively, involved conversational style unless the intrusion was egregious. Similarly, people who use a high-considerateness style would be more likely to perceive others as violating speaker rights if simultaneous speech occurs. We therefore use the number of perceived interruptions to divide our culture experts into high-considerateness and high-involvement subcultures. ${ }^{7}$

\section{STATISTICAL ANALYSIS AND RESULTS}

The means and standard deviations for both types of measurement are presented in Table 1 . The means represent the probability that any given utterance is judged to end in an interruption. The West-Zimmerman syntactic methods of measuring interruptions resulted in a lower probability of a transition being judged as an interruption (.0284) than any of the culture experts (whose average was .0713). This pattern supports Murray's contention that simple counting of syllables does not determine what an interruption is:

\footnotetext{
${ }^{7}$ It would have been preferable to use coders from different regions of the country to capture true subcultural effects, but we chose to use culture expert coders who would reflect the population of the interactants. We felt that use of the high-low difference would detect subcultural effects if any were present.
}

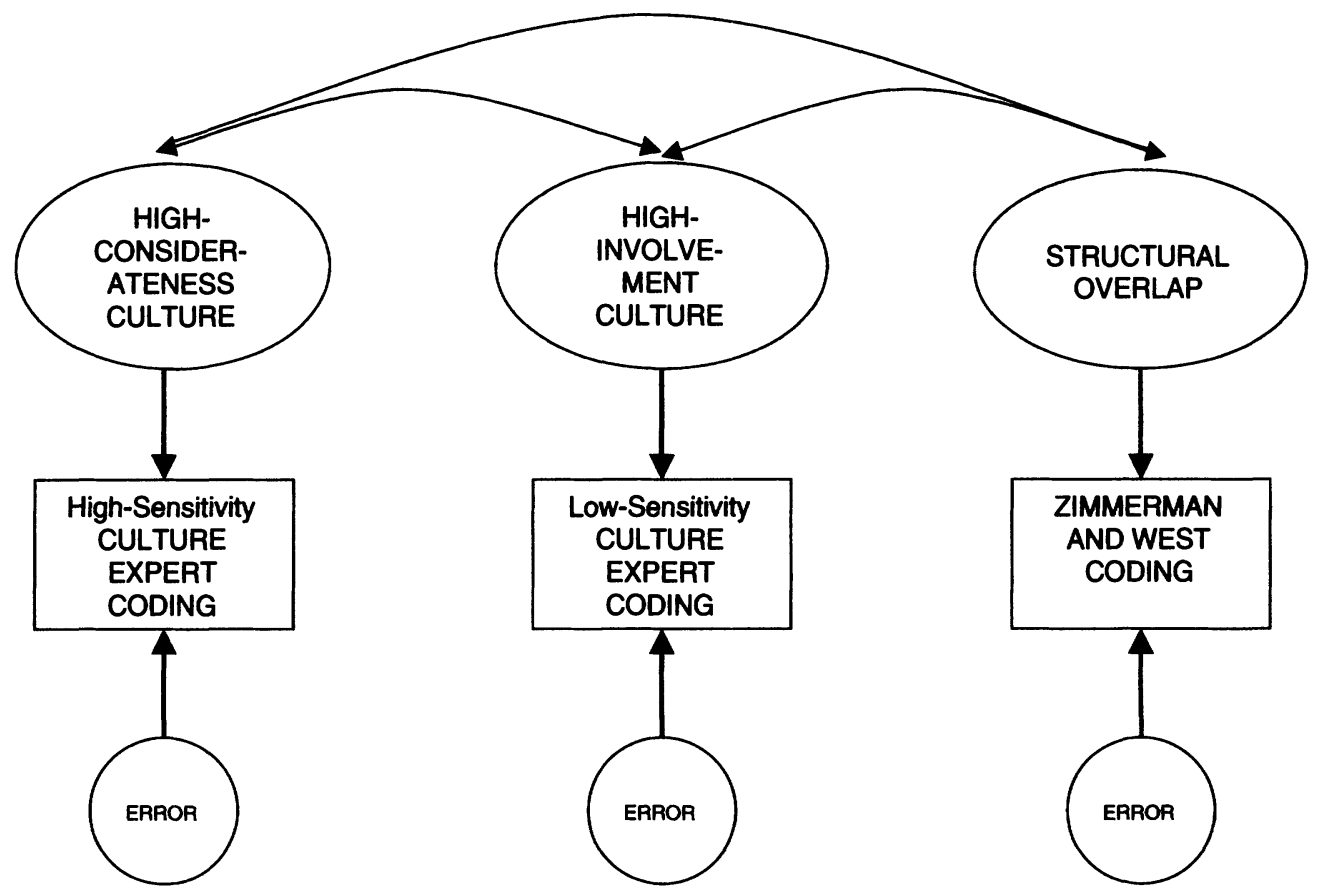

Figure 4. Sensitivity Model 
Table 1. Means and Standard Deviations for Culture Expert and West-Zimmerman Measurements for Interruptions

\begin{tabular}{lcc}
\hline \hline & Mean & SD \\
\hline Coder 1 (F) & .0787 & .2692 \\
Coder 2 (F) & .0508 & .2197 \\
Coder 3 (F) & .0600 & .2375 \\
Coder 4 (F) & .0812 & .2731 \\
Coder 5 (M) & .0495 & .2169 \\
Coder 6 (M) & .0926 & .2899 \\
Coder 7 (M) & .1054 & .3071 \\
Coder 8 (M) & .0522 & .2429 \\
WZ & .0284 & .1662 \\
\hline
\end{tabular}

Note: Letters in parentheses indicate whether the culture expert coder was male (M) or female (F).

the culture experts tended to take contextual variables into account and therefore identified more interruptions. The range of means for the culture expert coders, in which some coders identified roughly twice as many interruptions as others (.05 to .10), suggests the possibility of subcultural differences in how coders identify interruptions. This tendency to find more versus fewer interruptions, however, is not significantly correlated with the coder's gender. Male coders were slightly more likely than female coders to view a transition as an interruption (.0749 compared with .0677), but this difference is not statistically significant. In any case, we found substantial variability in the implicit process used by coders to label a transition as an interruption.

The average intercorrelation ${ }^{8}$ of our culture experts' judgments is .71. The females' judgments are correlated somewhat more closely with one another than the males' $(.73$ compared with .68), but not significantly so. The average correlation of the coders' judgments with the West-Zimmerman coding was .56 (again, slightly higher for females, at .59 , than for males, at .52). ${ }^{9}$

\footnotetext{
${ }^{8}$ Here and in our LISREL analyses which we present later, we use tetrachoric correlations, which are appropriate for the 0 and 1 forms of our data.

${ }^{9}$ An anonymous reviewer suggested that the gender of the interrupter and the interrupted speaker might influence the likelihood that culture experts labeled a speech act as an interruption. Orcutt and Mennella (1995), for example, found that "studentjudges" (similar to our culture experts) generally overestimated the amount of talk contributed by a woman who interrupted a man, whereas a man's estimated participation was not biased in conversations
}

Because our main interest in these data is determining the number of underlying constructs captured by our various measures, we begin with a traditional principal components analysis. Figure 5 shows the eigenvalues of the first six principal components. Using the traditional scree criteria, this solution clearly indicates a single factor underlying the nine measurements (West-Zimmerman syntactic measurement and the eight culture expert coders). The first eigenvalue is 3.517 ; all others are less than $1(.8925, .8177, .7980$, $.6781, .6348$, and so on).

Table 2 shows factor scores and communality estimates from this principal components analysis. Not surprisingly, the West-Zimmerman coding loads on the factor to a slightly lesser degree and has slightly lesser communality than do the eight culture expert codes. The syntactic measure is the only one that differs in method; the other eight share a common format (decisions by undergraduate coders using implicit criteria). Therefore we can expect that the West-

with a woman where he engaged in interruptions. Thus, in our study, culture experts might have coded males as having been interrupted more often than women, given gender biases about completion rights. The West-Zimmerman coding scheme is free from such biases because syllables are counted from a transcript by a coder who is blind to the speaker's sex.

To find out if culture experts displayed a gender bias in viewing completion rights, we regressed interruption variables created by each of the coding systems separately on interrupter's gender, interruptee's gender, and their interaction. The West-Zimmerman coding and the culture expert coding showed similar patterns of gender effects, although the gender effects were somewhat stronger in the West-Zimmerman coding. In particular, the culture experts were less likely than the West-Zimmerman coder to view opposite-sex exchanges as interruptions; this difference led to stronger gender main effects and a considerably stronger statistical interaction between speaker's gender and follower's gender in the analysis of the Zimmerman-West coded interruptions. When we separated the culture experts by gender, male culture experts were more likely to regard a speaker transition as anterruption if the original speaker was a male, while female coders showed no effect for speaker's gender. In this case, the male coders were more similar to the Zimmerman and West coder, an indication that the female coders might be assigned greater completion rights to female speakers. Individual coders varied considerably in their gender effects. In general, the study of contextual influences on the perception of interruptions appears to be a complex topic that deserves further study. 
Table 2. Factor Scores and Communality Estimates From Principal Components Factor Analysis

\begin{tabular}{lcc}
\hline & Factor Scores & Communality \\
\hline Coder 1 & .6377 & .4066 \\
Coder 2 & .6450 & .4160 \\
Coder 3 & .6728 & .4537 \\
Coder 4 & .5994 & .3593 \\
Coder 5 & .6781 & .3119 \\
Coder 6 & .6945 & .4824 \\
Coder 7 & .7361 & .5418 \\
Coder 8 & .5858 & .3432 \\
WZ & .4504 & .2028 \\
\hline
\end{tabular}

Zimmerman measure will be something of an outlier. Based on this principal components analysis, however, we would conclude that the nine indicators all measure the same underlying concept: that is, they represent a one-factor solution.

Basically we would interpret this result as indicating that people's sense of whether a transition constituted an interruption (presumably some type of disruption of normal turn-taking) is correlated so highly with West and Zimmerman's structural criteria for interruption that the two appear to indicate the same underlying phenomenon. We wish to explore further the measurement properties of these indicators, however. Therefore we proceed to a more sophisticated technique that allows us to examine the measurement structure in greater detail.

We performed a series of confirmatory factor analyses in LISREL8 (Jöreskog and Sörbom 1989). The LISREL8 models provide us with tests of the comparability of the measures. Jöreskog (1971) identified three levels for this comparability. The first of these is congeneric measurement, in which the measures of interest capture the same underlying

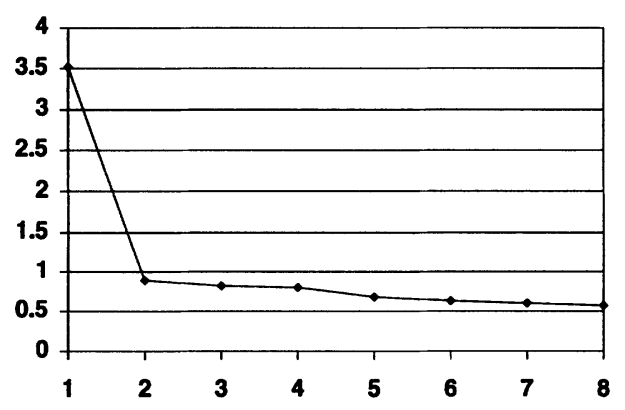

Figure 5. Eigenvalues for Principal-Components Analysis of Interruptions Indicators construct but do not necessarily do so in the same scale nor with the same amount of error. Tau-equivalent measures, the second level, capture the same underlying construct and do so in the same scale, but may not have the same amount of measurement error. If two measures capture the same underlying construct in the same scale, this means that each measure makes roughly the same contribution to the factor. In LISREL terminology, the parameters in the lambda matrix that relate the indicators to the underlying construct will be constrained to equivalence. Parallel measures, the third level, impose an additional constraint: they capture the same construct, do so in the same scale, and have the same amount of measurement error. Parallel measures are the most strongly equivalent indicators of an underlying construct; they can be substituted for one another.

In Table 3, we turn to the confirmatory factor analyses performed in LISREL. We present results for the congeneric, tau-equivalent, and parallel tests of the measures for a one-factor model (see Figures 1 and 2), ${ }^{10}$ the model that posits different underlying conceptions of interruptions by culture experts' gender (Figure 3), and the model that posits different underlying conceptions of interruptions for coders with high involvement and high considerateness, indicated in our data by high or low levels of identifying interruptions in the transcripts (Figure 4). ${ }^{11}$

When we compare rows in Table 3 , it is clear that the nine indicators of interruption

${ }^{10}$ In these LISREL8 analyses, we cannot test for the difference between a one-factor and a two-factor model because they would have the same degrees of freedom. One can either estimate a parameter in the lambda matrix that shows the relationship of the West-Zimmerman measure to the underlying construct of interruptions, or estimate the correlation between an underlying construct defined by the WestZimmerman measurement and an underlying construct defined by the culture experts' perceptions. The two are statistically equivalent and fit the data equally well.

${ }^{11}$ The four culture experts with the highest means of identified interruptions comprised the high-sensitivity group; the four cultural experts with the lowest means of identified interruptions comprised the lowsensitivity group (see Table 1 ). This split represents the largest gap in the distribution of means. 
Table 3. Confirmatory Factor Analysis for Comparison of Alternate Models

\begin{tabular}{lccc}
\hline \hline & $\begin{array}{c}\text { One- (or Two-) } \\
\text { Goodness of Fit }\end{array}$ & Factor & \\
\hline Congeneric & $4045.49(27)$ & $2686.81(25)$ & High vs. Low \\
Tau-Equivalent $^{\mathrm{b}}$ & $4823.87(35)$ & $3169.59(31)$ & $3599.71(25)$ \\
Parallel $^{\mathrm{c}}$ & $7154.86(44)$ & $4675.10(39)$ & $4055.17(31)$ \\
\hline
\end{tabular}

Note: The numbers in parentheses are degrees of freedom.

a The one- and two-factor models are indistinguishable because they have the same number of degrees of freedom. One can estimate the relationship between the West-Zimmerman measure and the culture expert factor either as a parameter relating the underlying construct to the observed measure (a parameter in the lambda matrix) or as a correlation between the two underlying constructs (a parameter in the phi matrix). Because there is only one measure of the West-Zimmerman structural approach, one can estimate a distinct two-factor model only by making the unrealistic assumption that the correlation between the underlying structural interruption construct and the underlying culture expert construct is zero.

${ }^{b}$ For the tau-equivalent and parallel models, lambdas (parameters indicating the relationships between the measures and the underlying constructs) are forced to be equal to one another within factors.

${ }^{c}$ For the parallel models, theta-deltas (the error variances) are forced to be equal to one another across all of the measures in one factor.

are not tau-equivalent or parallel in their relationship to the underlying construct. Nested models such as these can be compared by subtracting the chi-square value of the more restrictive model from that of the less restrictive model; the resulting value is a distributed chi-square with the degrees of freedom corresponding to the difference in degrees of freedom between the two models. For example, to assess whether the measures are tau-equivalent in the one-factor model, we subtract 4045.49 from 4823.87 (chi-square difference $=778.38$ ) and subtract 27 from 35 to obtain the corresponding degrees of freedom $(\mathrm{df}=8)$. We interpret the fact that the increase in chi-square is significant as an indication that the constraints in the tau-equivalent model (whereby the measures have the same relationship to the underlying construct in the lambda matrix) cause a significant decrease in the fit of the data to the model.

Table 3 shows clearly that the additional measurement constraints lead to a significant deterioration of fit in each of the models. In other words, we find no evidence that the two types of measures (culture expert and WestZimmerman) capture the same construct in the same scale (tau-equivalent) or with the same error (parallel). This result is not surprising, given our earlier observation that the West-Zimmerman measure produced a smaller loading on the first principal component (Table 2) and given the variation in the perceptual judgments made by the culture expert coders (Table 1). That said, the con- generic model fits the data moderately well, with a goodness of fit index of .91 , an adjusted goodness of fit index of .84 , and a normed goodness of fit index of .94 (Jöreskog and Sörbom 1989).

When we compare the columns of Table 3 , however, we find support for Murray (1985) and other researchers who argue for the subtleties of perception of interruption. Both models that specify different gendered perceptions of interruptions, and that allow for different perceptions by high-involvement and high-considerateness types, improve significantly on the simpler measurement model. In technical terms, when we release the constraints that force all eight coders to load on the same factor and allow them to load on two separate factors (while losing two degrees of freedom), the fit of the model to the data significantly improves. Because the gender culture model and the involvement/considerateness model are not nested, they cannot be compared directly; yet it is clear that differences by coder's gender are more significant than differences by the readiness with which a coder views an overlap as an interruption, even though the latter distinction was derived from the data themselves. (That is, the coders' means were used to classify them as high-involvement or highconsiderateness.) The congeneric gender culture model is the best-fitting model that we estimate; its parameters and fit statistics are displayed in Table 4. 
Table 4. Parameter Estimates for a Congeneric Confirmatory Factor Model With Three Factors

\begin{tabular}{cccc}
\hline \hline Construct & $\begin{array}{c}\text { Male } \\
\text { Interruption }\end{array}$ & $\begin{array}{c}\text { Female } \\
\text { Interruption }\end{array}$ & $\begin{array}{c}\mathrm{ZW} \\
\text { Interruption }\end{array}$ \\
\hline
\end{tabular}

Lambda Matrix Relating Underlying Constructs to Measures

Coder 1

Coder 2

1.0

Coder 3

1.04

Coder 4

1.06

0.95

Coder 5

.0

Coder 6

Coder 7

Coder 8

ZW

.0

$\begin{array}{ll}.0 & .0\end{array}$

.0

.0

.0

1.0

1.16
1.23

1.13

.0

.0

.0

.0

.0

.0

.0

.0

.0

1.0

Phi Matrix Relating Underlying Constructs to One Another

Male interruption

Female interruption

.66

.56

.51

.55

ZW interruption

.52

Notes: Theta Delta Matrix of Error Variances for Measures of Interruptions: Coder 1,.34; Coder 2,.28; Coder 3, .26; Coder 4,.40; Coder 5, .45; Coder 6, .26; Coder 7, .17; Coder 8, .31; ZW, 1.0. Goodness of Fit Statistics: ChiSquare with 25 Degrees of Freedom $=2686.81(P=.0)$; Goodness of Fit Index (GFI) = .93; Adjusted Goodness of Fit Index $(\mathrm{AGFI})=.88 ;$ Normed Fit Index $(\mathrm{NFI})=.96$.

We take our analyses to indicate that, although intuitive coders and the syntactic measurement of interruption are correlated highly enough to measure the same phenomenon by conventional measurement standards, different types of coders view interruptions in significantly different ways. These coders presumably are responding to different elements of the conversational context in deciding whether to label a given transition as an overlap.

This finding logically leads us to ask what types of cues are important in these perceptions. Although a thorough exploration of this topic is beyond the scope of this paper, we focus briefly on the criteria proposed by Murray (1985) for the severity of an intrusion. Following Murray's (1985) scale, we coded violations into four categories, with the most severe designated as 1 and the least severe as 4 . Severity was ranked by three undergraduate research assistants (not the same as the culture experts), with an intercoder reliability of .79 .

In Table 5, we show zero-order correlation coefficients to check the correctness of our hypothesis that more severe violations were coded as interruptions by more culture experts. We present correlations with the sum of all coders' judgments, and separate summed scores for male and female culture experts who coded a transition as an inter- ruption. The correlations between severity and the judgments of male culture experts, female culture experts, and all culture experts $(-.25,-.07,-.20)$ indicate that the more severe a violation, the more often coders rated it as an interruption. Our data support Murray's (1985) general point that more serious intrusions into another's speech-intrusions that interfere with a person's ability to express his or her ideas-are more likely to be considered interruptions.

The relationship is less strong (though still highly significant) for females than for males. In general, the gender cross-cultural communication perspective would predict that females would be more sensitive than men to relational aspects of interruptions; therefore this result is somewhat surprising. It may seem less perplexing if we consider that it may be evidence that women pay more attention than men to additional elements of the situational context. For instance, women might be influenced by factors such as the speakers' structural power positions, total floor time of speaker and interrupter, or the interrupter's intent (i.e., supportive or nonsupportive overlapping speech). Researchers have suggested that women are more collaborative and more relational in their speech patterns; thus the greater attention to contexts more varied than our simple operationalization of Murray's severity criterion 
Table 5. Correlation Coefficients for Number of "Culture Experts" Rating a Transition as an Interruption and the Severity of Interruption

\begin{tabular}{lcccc}
\hline \hline & $\begin{array}{c}\text { Number of } \\
\text { Males }\end{array}$ & $\begin{array}{c}\text { Number of } \\
\text { Females }\end{array}$ & Total & Severity \\
\hline Number of Males & 1.00 & & & \\
Number of Females & .27 & 1.00 & .00 & 1.00 \\
Total & .79 & -.07 & -.20 & 1.00 \\
Severity & -.25 & &
\end{tabular}

Notes: $N=2,368$. All correlations are significant at the .001 level.

would be consistent with the general argument.

\section{CONCLUSION}

Our results do not present a clear-cut answer to our original question: Do the two types of interruption measurements capture the same underlying construct? In a sense, the glass is both half full and half empty. The view that one wishes to adopt should almost certainly depend on the purposes of one's research.

On the one hand, the exploratory principal components analysis shows that the two measurement types load strongly on the same factor. By any conventional measurement standards, the different indicators are related closely enough to serve as measures of the same construct. We suggest that this result will be reassuring to the group process researchers who wish to code large bodies of discussion in terms of a variety of speech acts that might act as cues about status, power, or other social structures.

On the other hand, our results are considerably more complex for researchers who are focused more strongly on the meanings of conversations for their participants. The confirmatory factor results show that at least two categories of coders (by gender and by high or low considerateness) judge interruptions significantly differently. In other words, both the gender model and the high versus low model represent significant improvements on the fit of a simple model that holds all of the coders to be assessing the same underlying phenomenon. One suspects that other categorizations of the coders would lead to further improvements in fit. The subjective identification of an interruption varies significantly depending on who is doing the coding, although all coders generally are judging the same thing (according to the principal components analysis). This finding indicates caution for researchers who wish to study interruptions in terms of their meaning for participants, but also opens a fertile field for exploration. Clearly, the perception of interruptions is highly contextual and depends on subtle cultural features that are not yet clearly understood.

We offer our own first, small step in this direction with our analysis of gender. The gender difference in interruption perception is illuminated further by the finding that Murray's criteria for severity are less related to interruption identification for women than for men. This gender difference in identification of interruptions supports the cultural/socialization perspective: men and women seem to follow different assumptions and rules about interaction when interpreting talk. This is an interesting finding given that past research on interruption behavior found few gender effects (Aries 1996; James and Clarke 1988). In other words, men's and women's assumptions and rules do not translate into gender differences during interactions in a group setting. When determining speaker rights, however, men and women seem to attend to different aspects of the situation: women consider criteria other than Murray's severity principles. More in-depth studies of these perceptions and their basis will help to elaborate our understanding of gender cultures and to clarify how these cultures are translated into collaborative action.

\section{REFERENCES}

Albert, Ethel. 1964. “'Rhetoric,' 'Logic,' and 'Poetics' in Burundi." American Anthropologist 44:35-54.

Aries, Elizabeth. 1996. Men and Women in Interaction: Reconsidering the Differences. Oxford, UK: Oxford University Press. 
Atkinson, J. Maxwell and John Heritage. 1984. Structures of Social Action: Studies in Conversation Analysis. Cambridge, UK: Cambridge University Press.

Basso, Keith. 1974. "Basic Conversation Rules." Unpublished manuscript.

- 1979. Portraits of "The Whiteman": Linguistic Play and Cultural Symbols Among the Western Apache. Cambridge, UK: Cambridge University Press.

Beattie, Geoffrey W. 1980. "The Skilled Art of Conversational Interaction: Verbal and Nonverbal Signals in Its Regulation and Management." Pp. 193-211 in The Analysis of Social Skill, edited by William T. Singleton, Peter Spurgeon, and Robert B. Stammers. New York: Plenum.

Bennett, Adrian. 1981. "Interruption and the Interpretation of Conversation." Discourse Processes 4:171-88.

Carli, Linda L. 1989. "Gender Differences in Interaction Style and Influence." Journal of Personality and Social Psychology 56:565-76.

Chambliss, Catherine A. and Norah Feeny. 1992. "Effects of Sex of Subject, Sex of Interrupter, and Topic of Conversation on the Perceptions of Interruptions." Perceptual and Motor Skills 75:1235-41.

Cohen, Dov, Joseph Vandello, Sylvia Puente, and Adrian Rantilla. 1999. “'When You Call Me That, Smile!' How Norms for Politeness, Interaction Styles, and Aggression Work Together in Southern Culture." Social Psychology Quarterly 62:257-75.

Collier, Mary Jane. 1991. "Conflict Competence Within African, Mexican, and Anglo American Friendships." Pp. 132-54 in CrossCultural Interpersonal Communication, edited by Stella Ting-Toomey and Felipe Korzenny. Newbury Park, CA: Sage.

Covelli, Lucille H. and Stephon O. Murray. 1980. "Accomplishing Topic Change." Anthropological Linguistics 22:382-90.

Duncan, Starkey. 1972. "Some Signals and Rules for Taking Speaking Turns in Conversations." Journal of Personality and Social Psychology 23:283-92.

_. 1973. "Toward a Grammar for Dyadic Conversation." Semiotica 9:29-47.

Edelsky, Carole. 1981. "Who's Got the Floor?" Language in Society 10:383-421.

Fishman, Pamela M. 1983. "Interaction: The Work Women Do." Pp. 89-101 in Language, Gender, and Society, edited by Barrie Thorne, Cheris Kramarae, and Nancy Henley. Rowley, MA: Newbury House.

Garcia, Angela. 1991. "Dispute Resolution Without Disputing: How the Interactional Organization of Mediation Hearings
Minimizes Argument." American Sociological Review 56:818-35.

Gibson, David R. 1998. "Where Does Volubility Come From?" Presented at the International Conference on Social Networks, May 25, Sitges, Spain.

Goldberg, Julia. 1990. "Interrupting the Discourse on Interruptions: An Analysis in Terms of Relationally Neutral, Power-, and RapportOriented Acts." Journal of Pragmatics 14:883-903.

Goodwin, Marjorie Harness. 1980. "DirectiveResponse Speech Sequences in Girls' and Boys' Task Activities." Pp. 157-73 in Women and Language in Literature and Society, edited by Sally McConnell-Ginet, Ruth Borker, and Nelly Furman. New York: Praeger.

Hecht, Michael L., Mary Jane Collier, and Sidney A. Ribeau. 1993. African American Communication: Ethnic Identity and Cultural Interpretation. Newbury Park, CA: Sage.

Heritage, John and J. Maxwell Atkinson. 1984. "Introduction." Pp. ix-xvi in Structures of Social Action: Studies in Conversational Analysis, edited by J. Maxwell Atkinson and John Heritage. Cambridge, UK: Cambridge University Press.

James, Deborah and Sandra Clarke. 1988. "Women, Men, and Interruptions: A Critical Review." Pp. 231-80 in Gender and Conversational Interaction, edited by Deborah Tannen. New York: Oxford University Press.

Johnson, Cathryn. 1994. "Gender, Legitimate Authority, and Leader-Subordinate Conversations." American Sociological Review 59:122-35.

Jöreskog, Karl G. 1971. "Statistical Analyses of Sets of Congeneric Tests." Psychometrika 36:109-33.

Jöreskog, Karl G. and Dag Sörbom. 1989. LISREL 8: A Guide to the Program and Applications, 2nd ed. Chicago: SPSS.

Kennedy, Carol W. and Carl Camden. 1983. "A New Look at Interruptions." Western Journal of Speech Communication 47:45-58.

Kollock, Peter, Philip Blumstein, and Pepper Schwartz. 1985. "Sex and Power in Interaction: Conversational Privileges and Duties." American Sociological Review 50:34-46.

Lakoff, Robin. 1973. "Language and Woman's Place." Language in Society 2:45-79.

Lever, Janet. 1978. "Sex Differences in the Complexity of Children's Play and Games." American Sociological Review 43:471-83.

Maltz, Daniel N. and Ruth A. Borker. 1982. "A Cultural Approach to Male-Female Miscommunication." Pp. 197-216 in Language and Social Identity, edited by John 
J. Gumperz. Cambridge, UK: Cambridge University Press.

Maynard, Douglas W. and Steven E. Clayman. 1991. "The Diversity of Ethnomethodology." Annual Review of Sociology 17:385-418.

Murray, Stephen O. 1985. "Toward a Model of Members' Methods for Recognizing Interruptions." Language in Society 14:31-40.

- 1987. "Power and Solidarity in 'Interruption': A Critique of the Santa Barbara School Conception and Its Application by Orcutt and Harvey (1985)." Symbolic Interaction 10:101-10.

Murray, Stephen O. and Lucille H. Covelli. 1988. "Women and Men Speaking at the Same Time." Journal of Pragmatics 12:103-11.

Natale, Michael, Elliot Entin, and Joseph Jaffe. 1979. "Vocal Interruptions in Dyadic Communication as a Function of Speech and Social Anxiety." Journal of Personality and Social Psychology 37:865-78.

Octigan, Mary and Sharon Niederman. 1979. "Male Dominance in Conversations." Frontiers 4:50-54.

Okamoto, Dina and Lynn Smith-Lovin. 2001. "Changing the Subject: Gender, Status, and the Dynamics of Topic Change." American Sociological Review 66:852-73.

Orcutt, James D. and Diane L. Mennella. 1995. "Gender of Perceptions of Interruptions as Intrusive Talk: An Experimental Analysis and Reply to Criticism." Symbolic Interaction 18:59-72.

Reisman, Karl. 1974. "Contrapuntal Conversation in an Antiguan Village." Pp. 110-29 Explorations in the Ethnography of Speaking, edited by Richard Bauman and Joel Sherzer. Cambridge, UK: Cambridge University Press.

Sacks, Harvey, Emanuel Schegloff, and Gail Jefferson. 1974. "A Simple Systematics for Organization of Turn-Taking for Conversation." Language 50:696-735.

Schegloff, Emanuel. 2000. "Overlapping Talk and the Organization of Turn-Taking for Conversation." Language in Society 29:1-63.

Smith-Lovin, Lynn and Charles Brody. 1989. "Interruptions in Group Discussion: The Effects of Gender and Group Composition." American Sociological Review 54:424-35.

Smith-Lovin, Lynn and Dawn Robinson. 1992. "Gender and Conversational Dynamics." Pp. 122-57 in Gender, Interaction and Inequality, edited by Cecilia L. Ridgeway. New York: Springer-Verlag.
Stets, Jan and Peter Burke. 1996. "Gender, Control, and Interaction." Social Psychology Quarterly 159:193-220.

Strodtbeck, Fred L. and Richard D. Mann. 1956. "Sex Role Differentiation in Jury Deliberations." Sociometry 19:3-11.

Tannen, Deborah. 1984. Conversational Style: Analyzing Talk Among Friends. Norwood, NJ: Ablex. . 1986. That's Not What I Meant! How Conversational Style Makes or Breaks Your Relations With Others. New York: Morrow. 1990a. "Gender Differences in Topical Coherence: Creating Involvement in Best Friends' Talk." Discourse Processes 13: 73-90.

- 1990b. You Just Don't Understand: Women and Men in Conversation. New York: Morrow.

1994. Gender and Discourse. New York: Oxford University Press.

Thorne, Barrie and Zella Luria. 1986. "Sexuality and Gender in Children's Daily Worlds." Social Problems 33:176-90.

Tsoudis, Olga and Lynn Smith-Lovin. 1998. "How Bad Was It? The Effects of Victim and Perpetrator Emotion on Responses to Criminal Court Vignettes." Social Forces 2:695-722.

West, Candace. 1979. "Against Our Will: Male Interruptions of Females in Cross-Sex Conversation." Pp. 81-97 in Language, Sex, and Gender (Annals of the New York Academy of Sciences, 327), edited by Judith Orsanu, Mariam K. Slater, and Leonore Loeb Adler. New York: New York Academy of Sciences.

West, Candace and Angela Garcia. 1988. "Conversational Shift Work: A Study of Topical Transitions Between Women and Men." Social Problems 35:550-75.

West, Candace and Don Zimmerman. 1983. "Small Insults: A Study of Interruptions in CrossSex Conversations Between Unacquainted Persons." Pp. 103-17 in Language, Gender, and Society, edited by Barrie Thorne, Cheris Kramarae, and Nancy Henley. Rowley, MA: Newbury House.

Zimmerman, Don and Candace West. 1975. "Sex Roles, Interruptions and Silences in Conversations.” Pp. 105-29 in Language and Sex: Difference and Dominance, edited by Barrie Thorne and Nancy Henley. Rowley, MA: Newbury House. 
Dina Okamoto is assistant professor of sociology at University of California, Davis. Her research interests include identity, inequality, and race and ethnicity. Her most recent article "Changing the Subject: Gender, Status, and the Dynamics of Topic Change" (with Lynn SmithLovin) was published in American Sociological Review (v. 66, n. 6, 2001). Her current research explores panethnic identity formation and group behavior among Asian Americans.

Lisa Slattery Rashotte is assistant professor at The University of North Carolina at Charlotte. Her current research focuses on small group interaction, nonverbal behaviors, gender and expectations. She, Murray Webster, and Joe Whitmeyer are also currently studying the effect of second-order expectations on behavior.

Lynn Smith-Lovin is professor of sociology at the University of Arizona. She is a former chair of the American Sociological Association sections on the sociology of emotions and on social psychology. She served as president of the southern sociological society in 1999 and as co-editor of Social Psychology Quarterly from 1996-2000. She currently serves on ASA's Council. Her research focuses on identity, social interaction and emotion. Recent publications explore a relational analysis of changes in the discipline of sociology (in the September 1999 issue of Social Forces), the use of humor in group interaction (in the September 2001 Social Forces), and the status-organized nature of topic transitions in task group discussions (in the December 2001 American Sociological Review). She is currently beginning a National Science Foundationfunded study of how people manage the identities of their interaction partners. 